\title{
Beobachtungen des Halleyschen Kometen 1909 c
}

auf der Sternwarte des Collegio Romano in Rom von Prof. E. Millosevich, Dr. E. Bionchi und Dr. G. Zappa.

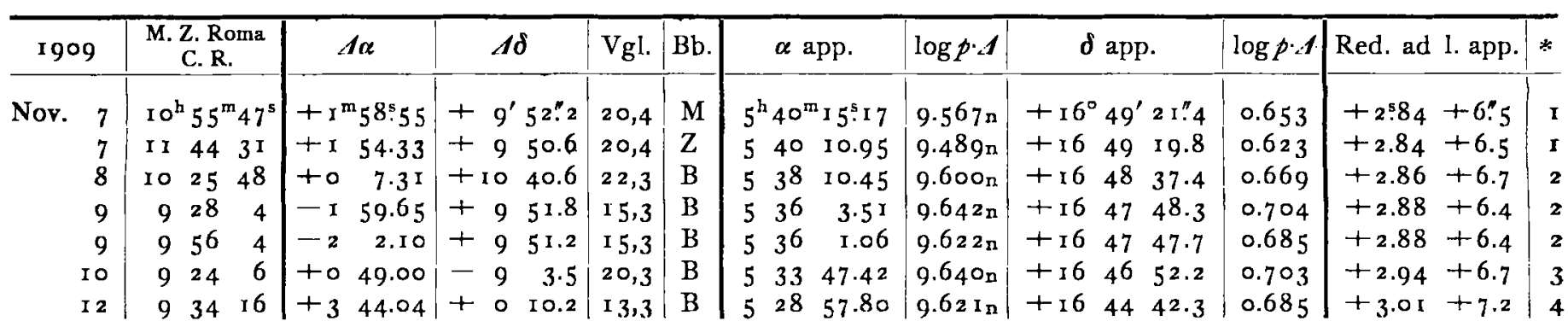

Nov. 7. L'astro ha nucleo di aspetto stellare di grandezza $\mathrm{I}^{\mathrm{m}} \cdot \mathbf{3}$, cioè ben più luminoso di grandezza $A$ e $B$ in A. N. 4359. La nebulosità dissimmetrica è debole, e si palesa al $38 \mathrm{~cm}$ quando l'astro ha un'altezza sopra $30^{\circ}$ (M.). Nov. 8. Cometa nebulare con tenue nucleo di $\mathrm{r}^{\mathrm{m}}{ }_{4}$. - Nov. 9. Confermo il carattere nebulare dell'astro che stimai di ${ }^{1} 3^{\mathrm{m}}$. Osservazioni difficili. - Nov. I2. Astro nebulare con accenno di nucleo.

Mittlere Örter der Vergleichsterne.

\begin{tabular}{|c|c|c|c|}
\hline * & $\alpha$ I 909.0 & $\delta \times 909.0$ & Autorität \\
\hline & $5^{\mathrm{h}} 3^{8^{\mathrm{m}}} 13^{\mathrm{s}} \cdot 78$ & $+16^{\circ} 39^{\prime} 22^{\prime \prime} 7$ & AG Berl A 167 I \\
\hline & $\begin{array}{lll}5 & 3^{8} & 0.28\end{array}$ & +163750.1 & $» \quad 1668$ \\
\hline
\end{tabular}

\begin{tabular}{|c|c|c|c|}
\hline * & $\alpha 1909.0$ & $\delta$ I 909.0 & Autorität \\
\hline 3 & $5^{\mathrm{h}} 32^{\mathrm{m}} 55^{\mathrm{s}} \cdot 4^{8}$ & $+16^{\circ} 55^{\prime} 49^{\prime \prime} \circ$ & AG Ber \\
\hline 4 & $\begin{array}{lll}5 & 25 & 10.75\end{array}$ & +164424.9 & Kü 2394 \\
\hline
\end{tabular}

\section{Beobachtungen von kleinen Planeten.}

Planet

(86) Semele (154) Bertha

(333) Badenia

(405) Thia

(460) Scania

I $909 \mathrm{HF}$

I $909 \mathrm{JA}$

\begin{tabular}{rr}
\multicolumn{2}{c}{ I 909} \\
Nov. & 15 \\
$》$ & 8 \\
$》$ & 5 \\
$》$ & 15 \\
$》$ & 10 \\
$》$ & 16 \\
$》$ & 15 \\
$》$ & 16
\end{tabular}

M. Z. Kopenh.

Gr.

I ${ }^{\mathrm{h}} 4 \mathrm{I}^{\mathrm{m}} 5^{6^{\mathrm{s}}}$

13 I $55^{8}$

13551

I 3 10 18

$\begin{array}{llll}10 & 2 & 47\end{array}$

$\begin{array}{lll}7 & 25 & 5\end{array}$

$10 \quad 28$ I 4

1027
I I ${ }^{\mathrm{m}} \cdot \mathbf{I}$

I I. 5

I 1.8

II.9

I 3.0

I 2.8

-

\begin{tabular}{|c|c|c|}
\hline \multicolumn{3}{|c|}{$\alpha$ app. } \\
\hline $3^{h}$ & $3^{2^{m}}$ & 225 \\
\hline I & & $48 \cdot 36$ \\
\hline 2 & 24 & 6.39 \\
\hline 3 & 48 & 26.17 \\
\hline I & 50 & 4.45 \\
\hline 1 & $5 \mathrm{I}$ & I 4.74 \\
\hline 3 & 40 & $49 \cdot 31$ \\
\hline & 39 & $51 \cdot 35$ \\
\hline
\end{tabular}

$\begin{array}{llll}3 & 39 & 5 & \mathbf{I} \cdot 35\end{array}$

$\log p \cdot \Delta$
$8.286_{n}$
$9.35^{2}$
9.171
8.975
$8.640_{n}$
9.027
$9.147 n$
$9.228 n$

$9.228 \mathrm{n}$

Kopenhagen, Universitäts-Sternwarte, rgog Nov. I 7 .

$\log p \cdot \Delta$

0.760

0.831

$0.73 \mathrm{I}$

0.649

0.799

0.829

0.742

0.749

C. F. Pechüle.

\section{Two new planets.}

\begin{tabular}{|c|c|c|c|c|c|}
\hline $\begin{array}{l}\text { Planet } \\
\left.\text { I } 909 \text { JA }^{1}\right)\end{array}$ & $\begin{array}{l}1909 \\
\text { Nov. } 7\end{array}$ & $\begin{array}{l}\text { Gr.m.t. } \\
\text { I } 3^{\text {h }} \text { I } 8^{\text {m.o }}\end{array}$ & $\begin{array}{c}\alpha \leq 909.0 \\
3^{h} 48^{m} 19: 0\end{array}$ & $\begin{array}{r}81909.0 \\
+19^{\circ} 15^{\prime} 11\end{array}$ & $\begin{array}{l}\mathrm{Mg} . \\
9^{\mathrm{m}} \cdot 5\end{array}$ \\
\hline ע & $\gg$ & I 246.0 & $\begin{array}{llll}3 & 46 & 31.9\end{array}$ & +1852.3 & $9 \cdot 5$ \\
\hline $909 \mathrm{JB}$ & Nov. 7 & i 455.0 & $\begin{array}{lll}3 & 57 & 57.7\end{array}$ & $+355^{8.5}$ & 10.0 \\
\hline$»$ & 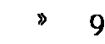 & 1437.0 & $\begin{array}{lll}3 & 55 & 56.8\end{array}$ & +3545.8 & Io. \\
\hline
\end{tabular}

Taunton, Mass., I 909 Nov. I3.

Foel H. Metcalf.

1) Vergl. die Auffindung in Königstuhl 1909 Nov. 9 in A. N. 183.16. $K b$.

New planet 1909 JC. (Telegramm von r 909 Dezember 2.) Planet November 30 i $2^{\mathrm{h}} 5^{\mathrm{m}} \cdot \mathrm{o}$ Greenwich $\mathrm{m}$. $\mathrm{t}$.

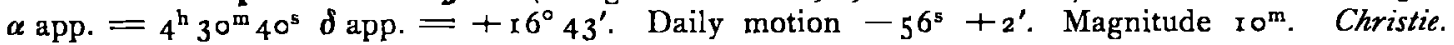

Berichtigung zu Nr. 4356 Band 182 pag. 192, Zeile 2 von unten 8 app. statt: I"6 lies: II"6, Red. ad 1. app. in 8 statt: 4"8 lies: 14 "8.

Inhalt zu Nr. 4373. W. Dziewulski. Über die periodischen Bahnen vom Hildatypus. 65. - W. Münch. Gelegentliche photometrische Beobachtungen einiger veränderlichen Sterne. 73. - H. H. Kritzinger. Notiz betreffend den großen roten Fleck auf Jupiter. 77. - E. Millosevich, E. Bianchi und G. Zappa. Beobachtungen des Halleyschen Kometen 1909 c. 79. - C. F. Pechüle. Beobachtungen von kleinen Planeten. 79. - F. H. Metcalf. Two new planets. 79. - W. H.M. Christic. New planet I 909 JC. 79. - Berichtigung. 79 . 